\title{
Anthropometric Digression in Hemifacial Microsomia
}

\author{
Astha Chaudhry ${ }^{1}$, Suma G.N. ${ }^{2}$, Puneeta Vohra ${ }^{3}$, Amwani Sood ${ }^{4}$ \\ 1, 2, 3, 4 Department of Oral Medicine and Radiology, Shree Guru Gobind Singh \\ Tricentenary (SGT) University, Gurugram, Haryana, India.
}

\section{INTRODUCTION}

Concurrent presence of auricular, ocular and facial anomalies is found in various overlapping conditions, one of which is Hemifacial Microsomia (HFM). German physician Carl Ferdinand Von Arlt first described HFM in 1881. ${ }^{1}$ Goldenhar described a triad of epibulbar choristomas, preauricular skin appendages in association with mandibular facial dysostosis, collectively called as the Goldenhar syndrome. Concurrent presence of vertebral anomalies along with Goldenhar triad is termed as Oculo-Auriculo-Vertebral dysplasia (OAV dysplasia) and OAV complex if microtia is also present. ${ }^{2}$

HFM affects one of every 5600 live births and is the second most common developmental craniofacial anomaly after cleft lip and palate. ${ }^{3}$ Males are more frequently affected, and the right side is more often affected than the left. ${ }^{4} \mathrm{HFM}$ is a syndrome of first and second branchial arches. The branchial arches are series of mesodermal thickenings in the wall of cranial most part of foregut with associated endodermal pouches and ectodermal clefts. It includes cartilage, skeletal element and nerve with each structure contributing to different organs of the body. The derivatives of first and second arch are shown in figure 1. Most of the first arch and few of the second arch derivatives show dysplasia in HFM depending on the grade of the disease.

Multiple classification systems have been proposed for the grading of the disease. Pruranzsky, Kaban and Madrid classification are basically the surgical classifications for the treatment of mandibular deformity. The most descriptive classifications are the SAT $^{5}$ and OMENS 6 systems. SAT describes the mandibular, auricular and nerve involvement. OMENS is more elaborative that takes into account the skeletal, external ear, neurological and soft tissue abnormalities. External ear abnormalities are peculiar with this condition and have been covered extensively in these classification systems. However, the middle ear and internal abnormalities seen with this condition have not been covered into any of these systems. Also, none of the classification systems describes any intraoral abnormalities.

The purpose of the present case report is to critically evaluate \& study the extent of involvement of various craniofacial structures in HFM with the help of various imageological and diagrammatic representations and to suggest the possible modifications in the existing system of classifications for comprehensive evaluation and grading of this pathology.
Corresponding Author: Dr. Astha Chaudhry, Flat No. 102, Tower G, Corona Optus, Sector 37C, Gurugram, Haryana-122001, India.

E-mail:dr.asthac@gmail.com

DOI: $10.14260 /$ jemds/2021/10

How to Cite This Article:

Chaudhry A, Suma G.N, Vohra P, et al. Anthropometric digression in hemifacial microsomia. J Evolution Med Dent Sci 2021;10(01):51-58, DOI: 10.14260/jemds/2021/10

Submission 06-08-2020,

Peer Review 22-10-2020,

Acceptance 29-10-2020,

Published 04-01-2021.

Copyright (C) 2021 Astha Chaudhry et al. This is an open access article distributed under Creative Commons Attribution License [Attribution 4.0 International (CC BY 4.0)] 


\section{PRESENTATION OF CASE}

A 10-year-old female patient with normal mental abilities reported to the department of Oral Medicine \& Radiology with a chief complaint of difficulty in eating food owing to irregularly arranged teeth \& inability to close the right eye on blinking. The patient was the first child of an 18 years old mother, with no history of consanguineous marriage, no significant family history or perinatal complication. The child was born with a marked facial asymmetry, malformed ear and a lateral facial cleft which was later closed surgically at the age of 3 months. A surgical scar was present extending from the right corner of the mouth to a line perpendicular to lateral canthus of the eye. The vital signs were normal.

On extra oral examination, head was dolichocephalic in shape with a convex profile and prominent forehead. On right side of the face, the hair line was receded about $20 \mathrm{~mm}$ cranially, right ear microtia \& atresia of external auditory meatus were present. The malformed ear was placed at a lower level than the contralateral side. The patient was unable to close the right eye on blink reflex additional to the upward rolling of the eye (positive bell's sign and phenomenon). Wrinkling of the skin of the forehead was absent on right side suggesting lower motor neuron facial palsy. There was reduction in the fullness of the cheek on right side suggesting hypoplasia of facial muscles and subcutaneous tissue. On further examination, hypoplastic right mandible \& maxilla, non-palpable temporomandibular joint, hypoplastic right masseter \& temporalis muscle were found. The mouth opening was $35 \mathrm{~mm}$. Intraorally, there was mixed dentition with severe malocclusion in the form of posterior crossbite on left side, buccal non-occlusion on right side, interdental spacing and a left side midline shift of $10 \mathrm{~mm}$. There were few decayed teeth, a high palatal arch and canting of occlusal plane. (Figure 2 and 3 ). The bulk of tongue was reduced on right side and it was seen deviated to the affected side at rest position as well as on protrusion. However, the tongue movements were normal. Some additional features included a high right buccal frenal attachment \& a tissue tag on posterior vestibular mucosa of the same side. (Figure 4).

An extensive imageological survey of the patient included panoramic view, water's view, Postero-Anterior (PA) skull view and computed tomography. The various mandibulofacial morphometric alterations were as follows:

\section{Skull}

There was hemi-microcephaly on the right side as measured anteroposteriorly on axial CT (Computed Tomography) scan (difference of $8 \mathrm{~mm}$ on both sides) and superoinferiorly as measured on PA skull from supraorbital margins (difference of $5 \mathrm{~mm}$ ). (Figure 5)

\section{Face}

There was marked facial asymmetry with reduced facial height $(9.4 \mathrm{~cm}$ vs. $10.5 \mathrm{~cm})$ and width $(6.3 \mathrm{~cm}$ vs. $7.2 \mathrm{~cm})$ on the right side. (Figure 5).

\section{Orbits}

There was slight alteration in the orbital inclination. However, no change in the size of orbital cavities was noted and innominate lines were normal. The interorbital distance was also normal $(2.5 \mathrm{~cm})$. (Figure 5)

\section{Nasal Cavity and Paranasal Sinuses}

The affected side nasal cavity was reduced in size and nasal floor was inclined superiorly towards the affected side. The frontal, ethmoidal and sphenoid sinuses showed reduced size on the affected side whereas the maxillary sinus was more or less similar. (Figure 5, 6, 10)

\section{Midface}

The right maxillary height was reduced. There was absence of right-side zygomatic arch and pterygomaxillary fissure. (Figure 6, 7)

\section{Mandible}

There was hypoplasia of right side mandibular body, angle, ramus, condylar and coronoid process. The ramus height and width was reduced $(3.0 \mathrm{~cm}$ and $2.5 \mathrm{~cm}$ respectively) on affected side as compared to normal side $(4.5 \mathrm{~cm}$ and $3.1 \mathrm{~cm}$ respectively) with steepening of the mandibular angle and a prominent antegonial notch as compared to nonaffected side. The depth of sigmoid notch was also reduced. There was canting of the occlusal plane superiorly on the right side. Another interesting finding was the presence of an enlarged follicular space enclosing mesioangularly inclined developing 47 \& tooth bud of 48 measuring approximately $5 \mathrm{~mm}$ from the coronal surface of 47 . The lesion was seen surrounded by corticated borders throughout. (Figure 7) Additionally, CT scan showed enlargement of mandibular foramen with normal lingula and a bony tubercle on the lateral surface of right ramus. (Figure 8,12 ) The section in soft tissue window revealed hypoplasia of parotid gland and masseter muscle on right side.

\section{Temporal bone}

The affected side showed absence of external auditory meatus and canal, absence of styloid process and incomplete pneumatization of the mastoid sinuses resulting in a smaller mastoid process. The petrous ridge of affected side temporal bone was seen at a lower level as compared to the contralateral side. (Figure 5, 9) There was hypoplasia of the glenoid fossa and articular eminence

\begin{tabular}{|cc|}
\hline First Arch & Second Arch \\
Mandible & Stapes \\
Maxilla & Styloid process \\
Incus & Stylohyoid ligament \\
Malleus & Facial nerve \\
External auditory meatus and middle ear cavity & Auricular muscles / external ear \\
Pharyngotympanic tube & Few of the facial muscles \\
Part of tongue & \\
Masseter and temporalis & \\
\hline Table 1. Derivatives of First and Second Arch Affected \\
in the Present Case \\
\hline
\end{tabular}

\section{Ear}

This structure is classically involved in HFM. Both the external and middle ear of right side showed marked alterations in the present case. There was complete atresia of right side external auditory canal and middle ear cavity. The middle ear ossicles (malleus, incus and stapes) were not appreciated on the affected side. The internal ear including the cochlea was normal. (Figure 9 and 10). On performing an audiogram, a severe conducting hearing loss ( $>40$ decibels) was revealed with respect to the right ear. 


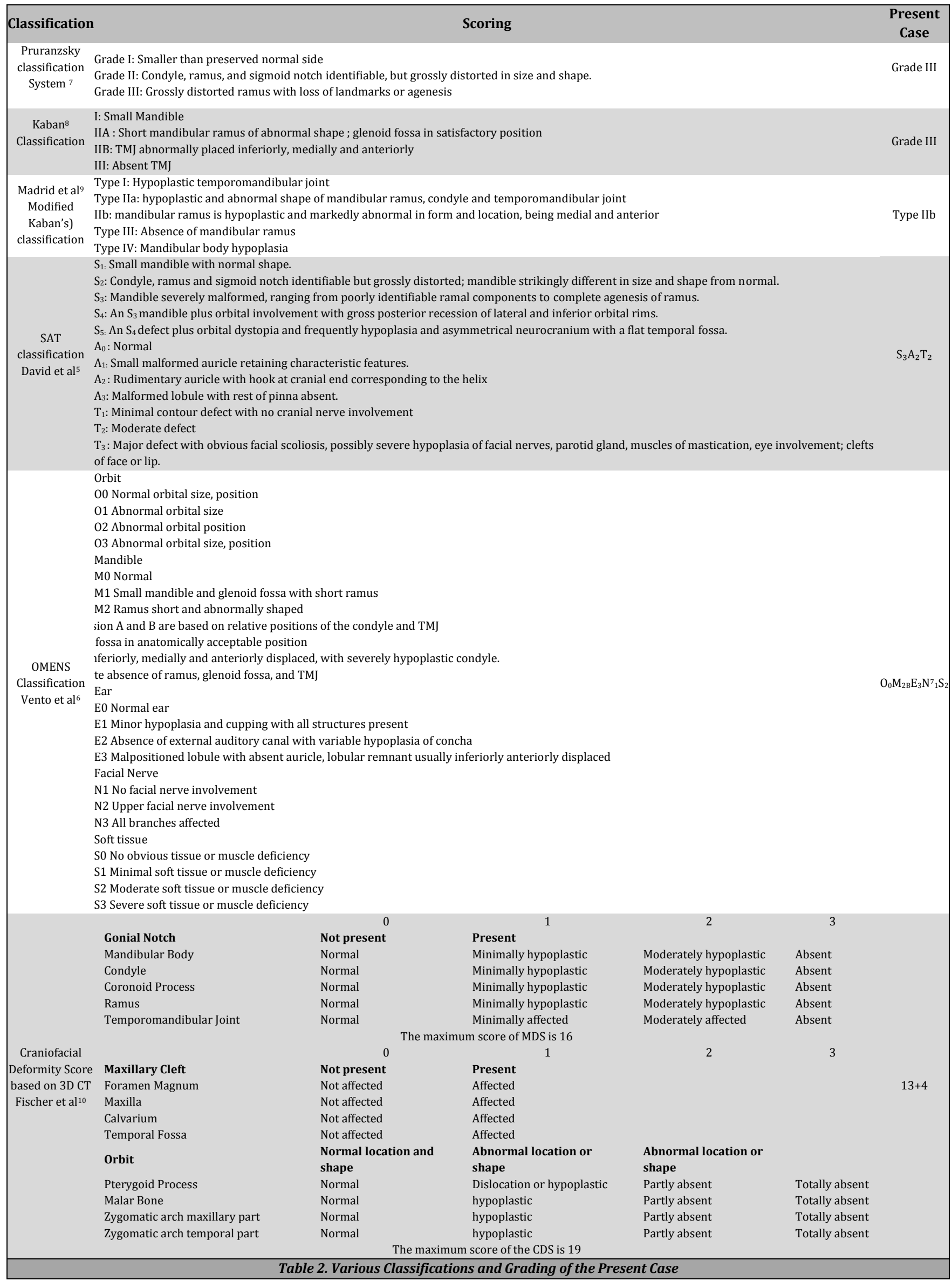




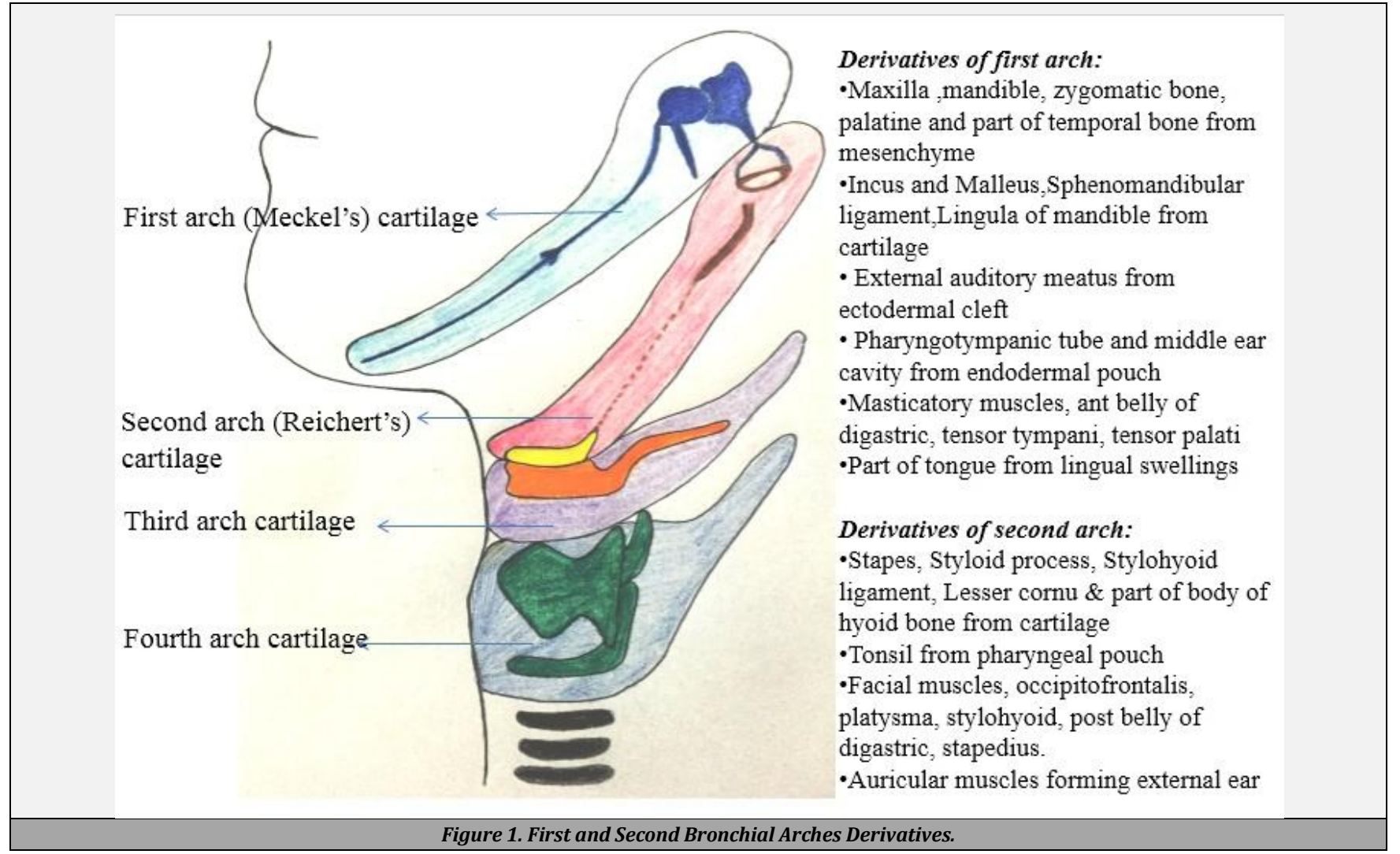
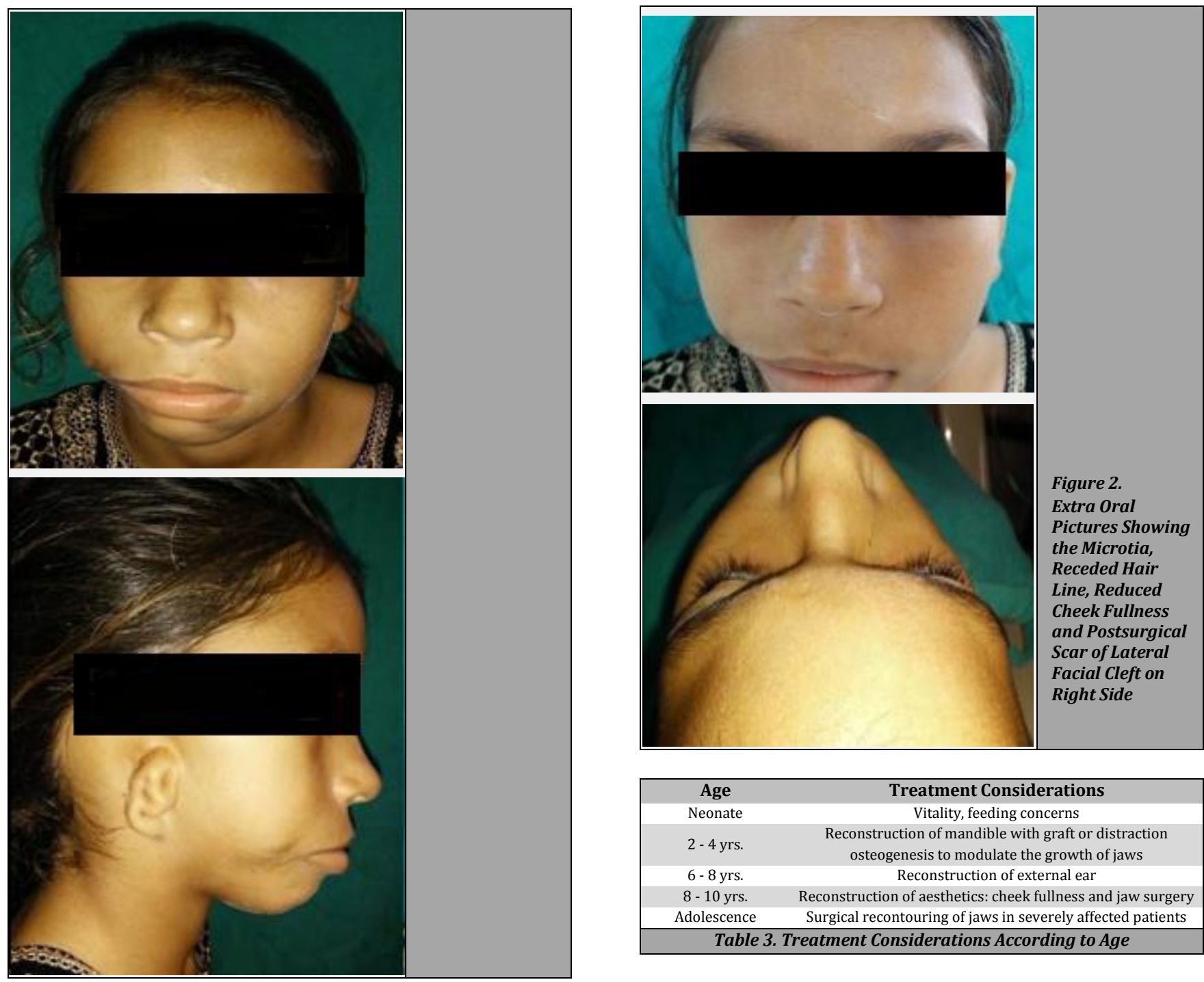

\begin{tabular}{|c|c|}
\hline Age & Treatment Considerations \\
Neonate & Vitality, feeding concerns \\
$2-4$ yrs. & $\begin{array}{r}\text { Reconstruction of mandible with graft or distraction } \\
\text { osteogenesis to modulate the growth of jaws }\end{array}$ \\
$6-8$ yrs. & Reconstruction of external ear \\
$8-10$ yrs. & Reconstruction of aesthetics: cheek fullness and jaw surgery \\
Adolescence & Surgical recontouring of jaws in severely affected patients \\
\hline Table 3. Treatment Considerations According to Age \\
\hline
\end{tabular}



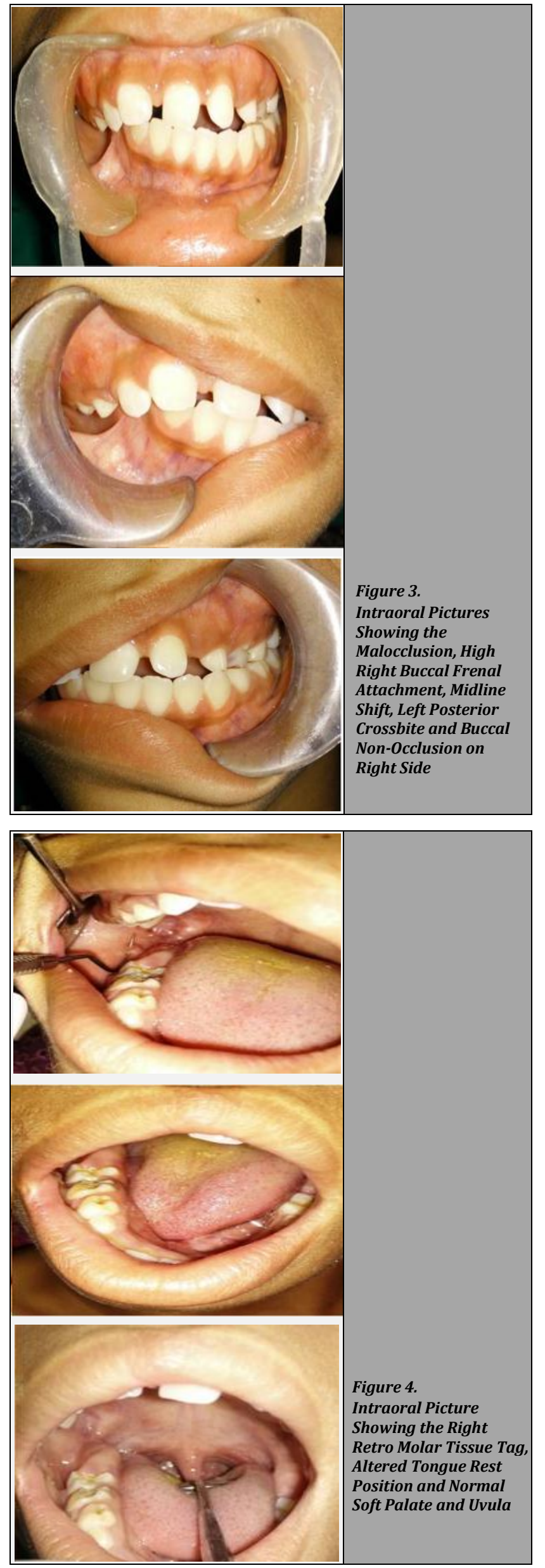
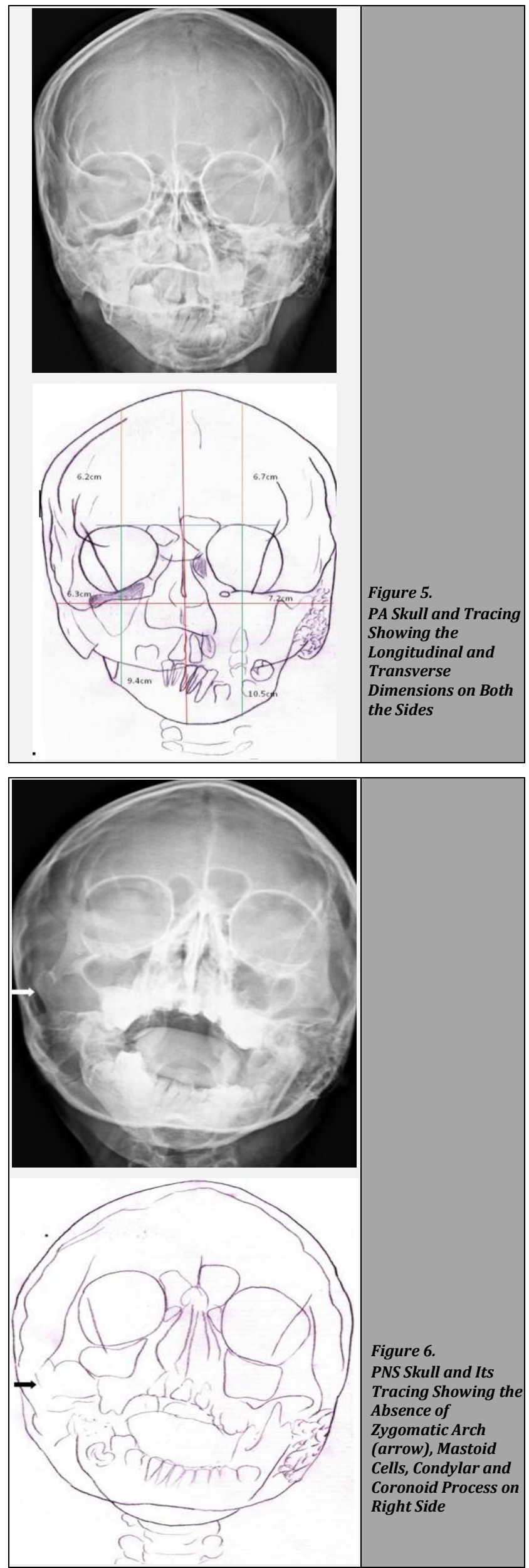

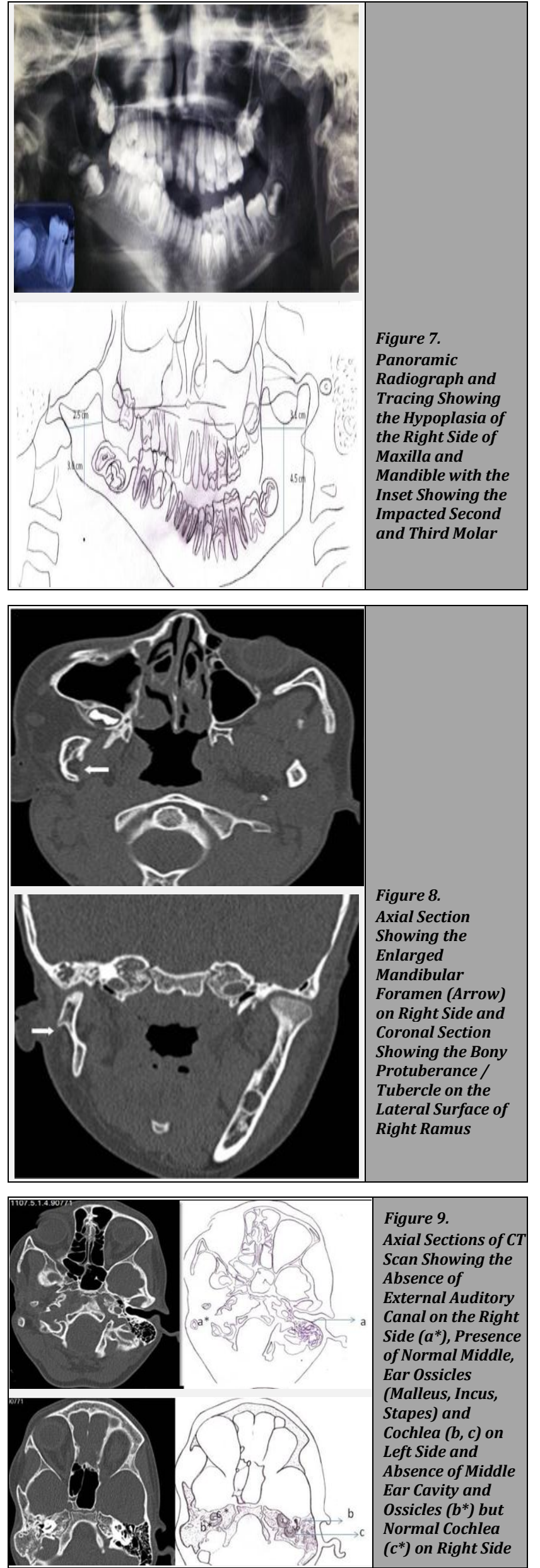
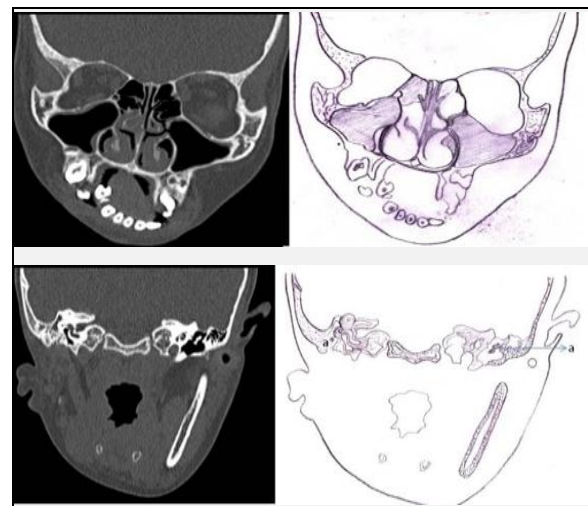

Figure 10.

Coronal CT Section

Showing the

Altered Inclination

of Head and Nasal

Cavity, Lower

Placement of

Rudimentary Right

External Ear,

Normal Middle Ear

Cavity and Ossicles

(a) on Left Side and

Absence of the

Same on Right Side $\left(a^{*}\right)$
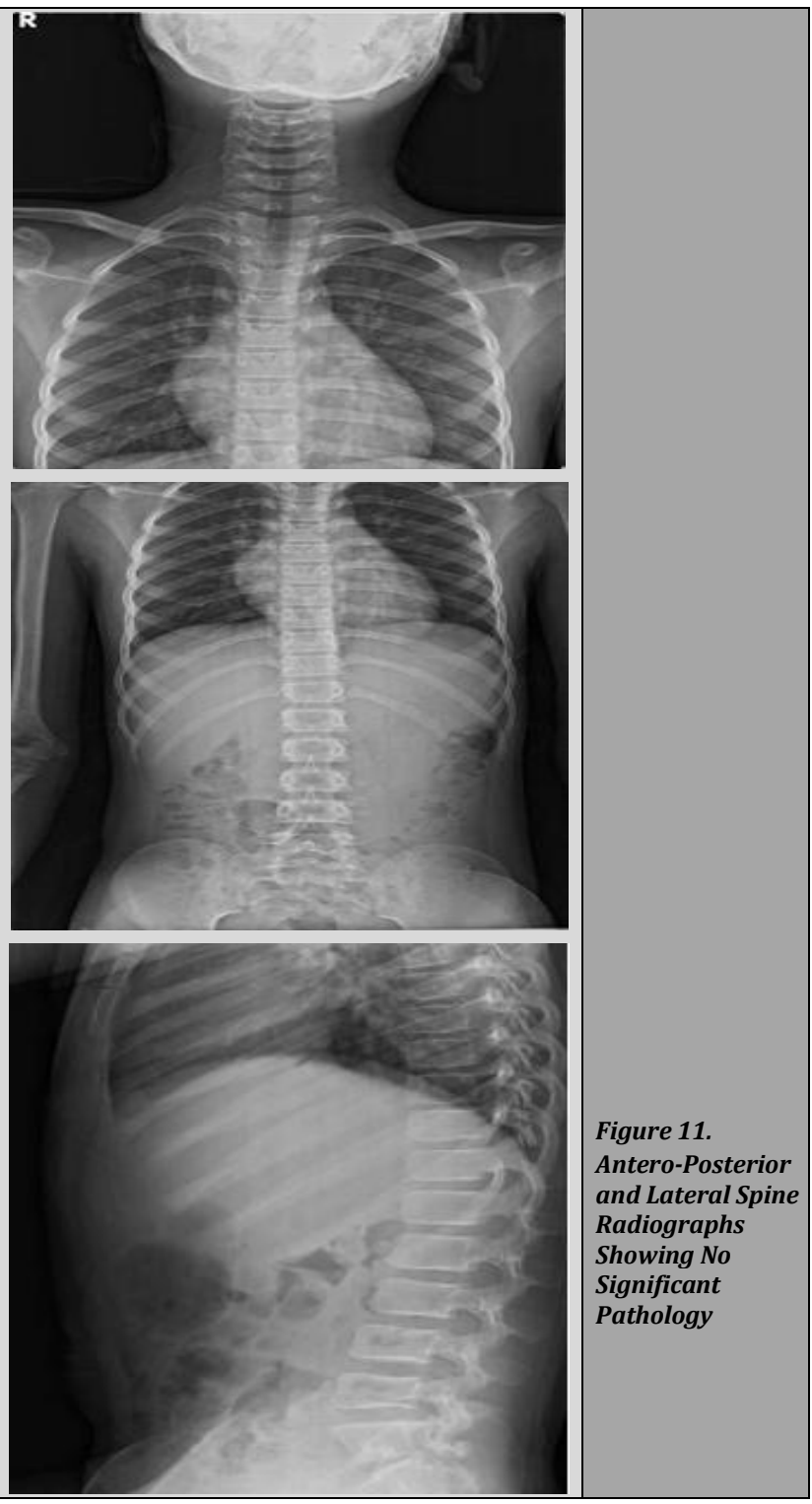

No appreciable deformity was found on x-ray chest \& spinal x-ray of cervical, thoracic, lumbar \& sacral vertebrae thus ruling out Goldenhar syndrome. (Figure 11)

The blood investigations \& the ECG (Electro-Cardio-Gram) revealed normal findings. There was no abnormality associated with the hyoid bone and tonsil. Hence, few of the first arch and second arch derivatives were affected in this patient. (Table 1). Multiple classifications systems have been proposed in order to justify the varied range of clinical \& radiological manifestations of this disease. The grading of the 
present case according to various classifications is given in Table 2. The patient was advised surgical treatment in the form of distraction osteogenesis to favour the growth of hypoplastic mandible followed by orthodontic treatment.
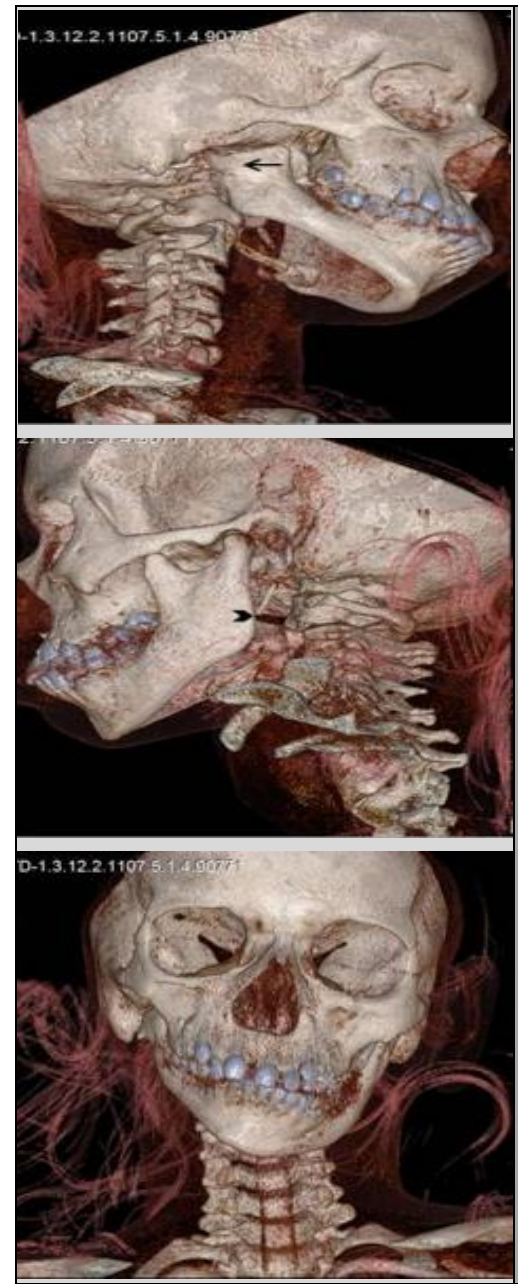

Figure 12.

3D Reconstruction Showing the Hypoplasia of the Right-Side Mandible and Zygoma and Normal Left Side. The Arrow also Points the Tubercle on the Lateral Surface of Right Ramus. Arrowhead Showing the Normal Styloid Process on Left Side and Absence of Same on Right Side
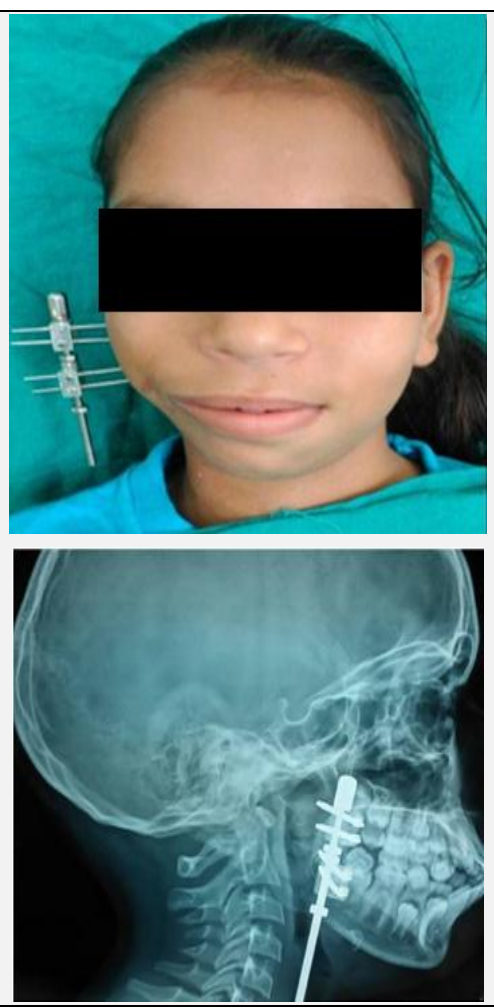

Figure 13.

Post-Operative Picture and Radiograph Showing Distraction Device in Distracti
Place
Distraction surgery was performed using extraoral approach and the distraction device was screwed open after regular intervals. A distraction of $0.5 \mathrm{~mm}$ was done 12 hourly, for 3 months and continuous follow up is maintained. (Figure 12).

\section{DISCUSSION}

Facial asymmetry is the hallmark of HFM which may not be appreciable in infancy, but becomes evident usually by the age of 4 yrs. The facial hypoplasia may be predominantly vertical or transverse or mixed with greatest degree of hypoplasia seen along the line from the residual ear till angle of mouth. ${ }^{2}$ The present case showed mixed vertical and transverse hypoplasia. The mandibular hypoplasia accounted for much of the asymmetry. The ramus was more hypoplastic than body thus acquiring a steep slope. This led to the lateral rotation of the lower jaw and posterior displacement of mandibular angle.

Corresponding to the skeletal deficiencies of maxilla, zygoma and mandible, there was reduced mass of muscles involved. Hence, the deficiency of right masseter and temporalis was easily explained. Only the muscle bulk was reduced, the movements were normal. Also, the sensory supply of the face was normal suggesting that trigeminal nerve was not affected.

Preauricular skin tags are common in HFM and may be seen between ear and angle of mouth, may be sessile or pedunculated, unilateral or bilateral or may also be present at aberrant site like retroauricular, nostril, nose tip, and eyelid. Facial pits and sinuses may also be seen with or without skin tags. In our case, preauricular skin tag and tissue tag in the retromolar region of the affected side was seen. The intraoral occurrence of aberrant tissue tags has not been reported till now in HFM and hence, is a peculiar finding with the present case.

In the oral cavity macrostomia was present initially secondary to transverse facial cleft. Tongue showed reduced bulk and an altered position at rest and at protrusion. The tongue also develops partly from first arch tissues and thus, showed reduced bulk. However, the altered position at rest and on protrusion was secondary to hypoplasia and hypoglossal nerve paralysis as rest of the tongue movements were normal. High buccal frenal attachment was also noted. The outgrowth of parotid gland arises in relation to the line of fusion of maxillary and mandibular processes for the formation of cheek. This abnormality of fusion as depicted by lateral facial cleft explains the hypoplasia of the parotid gland. The present classification systems did not take into account any of the intraoral features like mucosal tags, abnormal frenal attachments, malocclusion etc which should be considered.

Ear was classically involved in the present case. There was microtia and absent external auditory canal. This is due to abnormal development of auricular hillocks ${ }^{11}$ and proportional to this was the conduction hearing loss. The middle ear cavity was stenosed. There was fusion of malleus and incus. The inner ear was normal. Facial palsy was seen in this case secondary to the microtia and abnormal middle ear. External ear abnormalities are extensively covered in SAT and OMENS classification. However, the abnormality in middle ear and normal internal ear as seen in the present case did not fit into any of the classifications. Hence, we propose a 
modification in the existing OMENS classification to include middle ear abnormalities along with external ear pathology as a severe grade E4. As internal ear abnormalities are seen with only $6 \%$ of the cases, the presence of internal ear pathology along with external and middle ear should be given a severe grade of E5. Also, audiologic abnormalities have not been considered in the classification systems. Cousley ${ }^{12}$ has proposed to add an asterisk to the E component of acronym OME*NS to indicate the type and degree of hearing loss which has been supported by Rahbar et al $^{13}$ when they found the conductive, sensorineural and mixed type of hearing abnormalities in 40 HFM patients.

Many theories regarding the aetio-pathogenesis of HFM have been proposed based on embryologic, clinical and laboratory studies. A widely accepted theory for the pathogenesis of HFM is the disruption in the normal development of the first and second arch derivatives due to the haemorrhage associated with the formation of the stapedial arterial system during embryogenesis. Also, the impaired interaction between neural crest cells and branchial arch mesenchyme and mutations in the Msx class of homeobox genes that are expressed in neural crest cells may be responsible. Studies have suggested that an early loss of neural crest cells may be responsible for the clinical presentation of HFM. Additional problems, such as cleft palate (seen in as many as 10 percent of the cases) and cardiac anomalies (seen in as many as 50 percent of the cases) associated with HFM are also related to an early loss of neural crest cells. The degree of severity of facial deficiency is determined by the extent of loss of neural crest cells and thus, this dictates the severity of the clinical presentation. ${ }^{2,14}$

The management of these patients aims in the correction of functional and aesthetic value. 15 Due to growth and development issues, management is tailored according to the age and functional requirements as given in table 3 . In the present case, a multidisciplinary approach was followed which included opinion and consultation from otolaryngologist, paediatrician, orthodontist and oral and maxillofacial surgeon who repaired the lateral facial cleft early in age to maintain the feeding and distraction osteogenesis at present age to promote the growth of mandible. The patient is on continued follow up.

\section{CONCLUSION}

Hemifacial microsomia is a variable asymmetric craniofacial malformation resulting in hypoplasia of the components of the first and second branchial arches. Systemic evaluation should include complete audiologic evaluation, cardiovascular, orthopaedic, maxillofacial evaluation and computed tomography of the maxillofacial and temporal region. Of all the classification systems OMENS is comprehensive and also grades the disease. However, it does not take middle and internal ear abnormalities, audiologic alterations and intraoral pathologies into consideration. Hence, we propose to add 2 more grades for evaluation of ear in existing OMENS classification to satisfactorily grade this enigmatic pathology.
Also, a new classification to grade the intraoral abnormalities is required.

Financial or other competing interests: None.

Disclosure forms provided by the authors are available with the full text of this article at jemds.com.

\section{REFERENCES}

[1] Kalsotra P, Chowdhary A, Bhagat DR, et al. Craniofacial Microsomia. J K Sci 2006;8(3):168-70.

[2] Som P, Curtin H. Head and neck imaging. $5^{\text {th }}$ edn. St. Louis: Mosby 2011: p. 3080.

[3] Monahan R, Seder K, Patel P, et al. Hemifacial microsomia. Etiology, diagnosis and treatment. J Am Dent Assoc 2001;132(10):1402-8.

[4] Kapur R, Kapur R, Sheikh S, et al. Hemifacial microsomia: a case report. JISPPD 2008;26(5):34-40.

[5] David DJ, Mahatumarat C, Cooter RD. Hemifacial microsomia:a multisystem classification. Plast Reconstr Surg 1987;80:525-33.

[6] Vento AR, Labrir RA, Mulliken JB. The O.M.E.N.S, classification of hemifacial microsomia. Cleft Palate Craniofacial J 1991;28(1):68-76.

[7] Pruzansky S. Not all dwarfed mandibles are alike. Birth Defects 1969;5:120-9.

[8] Kaban LB, Moses MH, Mulliken JB. Surgical correction of hemifacial microsomia in the growing child. Plast Reconstr Surg 1988;82(1):9-19.

[9] Madrid JRP, Montealegre G, Gomez V. A new classification based on the Kaban's modification for surgical management of craniofacial microsomia. Craniomaxillofac Trauma Reconstr 2010;3(1):1-7.

[10] Fischer CEH, Zonneveld FW, Vaandrager JM, et al. CTbased size and shape determination of the craniofacial skeleton: a new scoring system to assess bony deformities in hemifacial microsomia. J Craniofac Surg 2001;12(1):87-94.

[11] Sze RW, Paladin AM, Lee S, et al. Hemifacial microsomia in pediatric patients: asymmetric abnormal development of the first and second branchial arches. American Journal of Roentgenology 2002;178(6):1523-30.

[12] Cousley RR. A comparison of two classification systems for hemifacial microsomia. Br J Oral Maxillofac Surg 1993;31(2):78-82.

[13] Rahbar R, Robson C, Mulliken J, et al. Craniofacial, temporal bone and audiologic abnormalities in the spectrum of hemifacial micorsomia. Arch Otolaryngol Head Neck Surg 2001;127(3):265-71.

[14] Madi M, Shetty SR, Babu SG, et al. Hemifacial microsomia: a case report and overview. Cukurova Medical Journal 2014;39(3):625-35.

[15] Romsee MC, Verdonck A, Schoenaers J, et al. Treatment of hemifacial microsomia in a growing child: the importance of co-operation between the orthodontist and the maxillofacial surgeon. J Orthod 2004;31(3):190-200. 